\title{
Simulation of lateral color for a hybrid refractive-diffractive eyepiece by field tracing methods
}

D. Batte, M. Kuhn, F. Wyrowski

D. Batte, M. Kuhn, F. Wyrowski, "Simulation of lateral color for a hybrid refractive-diffractive eyepiece by field tracing methods," Proc. SPIE 9793, Education and Training in Optics and Photonics: ETOP 2015, 979300 (8 October 2015); doi: 10.1117/12.2223080

SDIE Event: Education and Training in Optics and Photonics: ETOP 2015, 2015, Bordeaux, France 


\title{
Simulation of Lateral Color for a Hybrid Refractive - Diffractive Eyepiece by Field Tracing Methods
}

\author{
D. Batte, Scoptique, Bordeaux, France \\ M. Kuhn, LightTrans GmbH, Jena, Germany \\ F. Wyrowski, Friedrich-Schiller-University, Jena, Germany
}

\begin{abstract}
The simulation of optical systems with both, refractive and diffractive components is very challenging and can neither be solved by a classical ray tracing approach nor by a general rigorous Maxwell solver. That is why the concept of field tracing has been introduced which is a generalization of ray tracing: harmonic fields are traced through the optical system instead of ray bundles. This allows the smooth combination of different modeling techniques in different subdomains of the system called unified optical modeling. The techniques can be adapted locally to minimize the numerical effort whereas the accuracy is as high as needed. In this paper we apply field tracing techniques for the simulation of an eyepiece containing a diffractive lens for minimizing the lateral color. The results presented include higher diffraction orders appearing due to the grating-like structure of the diffractive lens.
\end{abstract}

Keywords: field tracing; ray tracing; diffractive lens; geometrical optics; physical optics; system modeling; lateral color

\section{INTRODUCTION}

The development and design of optical systems can be enhanced considerably by using simulation and design tools. Thereby several challenges occur since all components of the system have to be modeled with sufficient accuracy. This includes light sources, optical components and detector functions. Often conventional optical components, such as lenses and prisms, are combined with micro- and nanostructured components. Depending on the application several physical effects including polarization, refraction and diffraction, spatial and temporal coherence may have to be taken into account. The sizes of structures appearing in the system strongly influence the performance of different numerical simulation techniques. The ultimate goal is to solve Maxwell's equations for the system at hand. However, general solvers as for example Finite Element Methods are not feasible with respect to the required computer resources in most cases due to the lateral extension of the system. On the other hand, classical ray tracing is fast but is not accurate enough for the simulation of most micro- and nanostructured components.

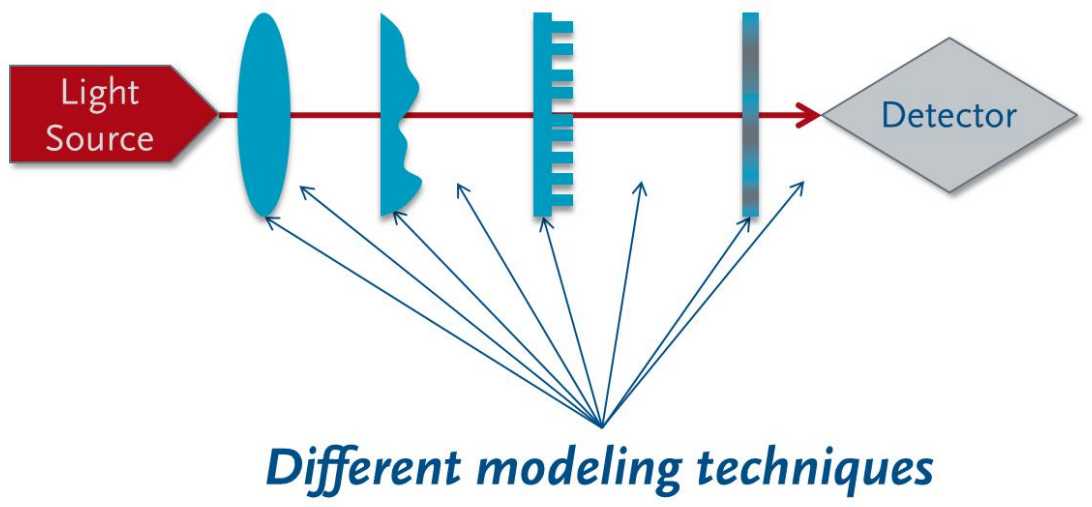

Figure 1: Usage of different propagation techniques adapted to the structural properties of the optical components.

Starting from this observation the concept of field tracing has been developed [1]. It allows a smooth combination of different simulation techniques in order to simulate an optical system as fast as possible and as accurate as required. Suitable modeling techniques are selected for different components of a system, e.g. geometric field tracing for

Education and Training in Optics and Photonics: ETOP 2015, edited by Eric Cormier, Laurent Sarger Proc. of SPIE Vol. 9793, 979300 · @ 2015 SPIE, IEEE, OSA, ICO · doi: 10.1117/12.2223080 
propagating a laser beam through a lens, the Rayleigh Sommerfeld integral to obtain the beam in the focus of the lens and FMM (Fourier Modal Method) to model the scattering of a beam at some grating. In order to combine such methods a common representation of the light is required. In field tracing, electromagnetic harmonic fields are traced through the system. This approach provides three fundamental advantages of practical concern [1]: (1) field tracing enables unified optical modeling. Its concept allows the utilization of any modeling technique that is formulated for vectorial harmonic fields in different subdomains of the system. (2) The use of vectorial harmonic fields as a basis of field tracing permits a great flexibility in source modeling. By propagating sets of harmonic field modes through the system, partially temporally and spatially coherent light as well as ultrashort pulses can be investigated [2]. (3) In system modeling and design, the evaluation of any type of detector function is crucial. The use of vectorially formulated harmonic fields provides unrestricted access to all field parameters and therefore it allows the introduction and evaluation of any type of detector.

In this paper we show the basic principles and equations of field tracing. We apply the approach for the analysis of an eyepiece containing a diffractive lens. This lens is used in order to compensate the lateral color of the system.

\section{FIELD TRACING METHODS}

In order to discuss the field tracing equations we assume that the optical system is located in the domain $\Omega \subset R^{3}$. Each optical component is represented by a subdomain $\Omega_{j}$ in which the refractive index $\hat{n}(r)$ with $r=(x, y, z)$ is inhomogeneous. We denote the boundaries of $\Omega_{j}$ by $\Gamma_{j}$. We assume that all $\mathrm{J}$ subdomains are embedded in the homogeneous dielectric with refractive index $n$.

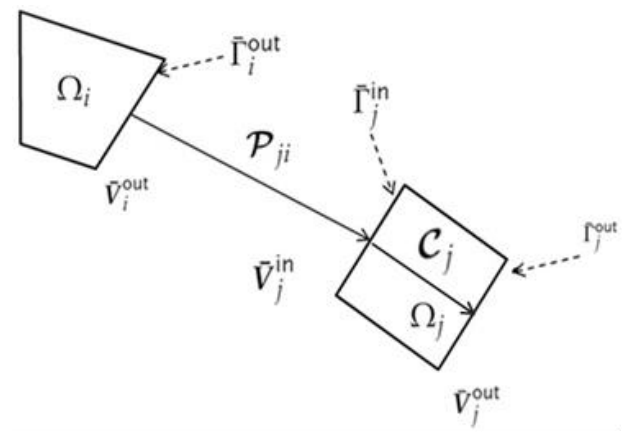

Figure 2. Formally a system is subdivided into J subdomains $\Omega_{j}$. All of the subdomains are embedded in a homogeneous and isotropic medium of refractive index $\mathrm{n}$. The boundaries of the subdomains are denoted by $\Gamma_{j}$.

We denote the field at the boundary $\Gamma_{j}$ by: $V_{j}=V\left(r \in \Gamma_{j}\right)=\left(E_{x}, E_{y}, E_{z}, B_{x}, B_{y}, B_{z}\right)$. Note, in practice we compute $E_{x}$ and $E_{y}$ by propagation. The other components of the electromagnetic field can be calculated on demand from the Maxwell's equations.

Let us look at the modeling problem for the subdomain $\Omega_{j}$ illustrated by Figure 2 . The component is hit by the input field $V_{j}^{\text {in }}$. The input field is mapped to the output field $V_{j}^{\text {out }} \mathrm{j}$ by the operator $C_{j}$ by

$$
\boldsymbol{V}_{j}^{\text {out }}=\boldsymbol{C}_{j} \cdot \boldsymbol{V}_{j}^{\text {in }}
$$

The operator $C_{j}$ models the effect of the component inside the subdomain $\Omega_{j}$ on the input field and delivers the outgoing field due to this effect by neglecting all other inhomogeneities $\Omega_{i}$ for $i \neq j$. The input field of subdomain $\Omega_{i}$ results from the output fields of the other subdomains. Thus, we define the operator $P_{j i}$ which maps 
the output field of subdomain $\mathrm{j}$ to the incident field of subdomain $\Omega_{i}$ with $i \neq j$ :

$$
\boldsymbol{V}_{j}^{\text {in }}=\mathcal{P}_{j i} \cdot \boldsymbol{V}_{i}^{\text {out }}
$$

This operator propagates the fields through the homogeneous dielectric in which all subdomains are embedded. Now we assume that we have a single source $V_{j}^{\text {source }} \neq 0$ for $j=1$ only and that the source field propagates through the components in the sequence of the index numbers. For $j>1$ we obtain

$$
\boldsymbol{V}_{j}^{\text {out }}=\boldsymbol{C}_{j} \cdot \mathcal{P}_{j, j-1} \cdot \boldsymbol{V}_{j-1}^{\text {out }}
$$

and $V_{1}^{\text {out }}=V_{1}^{\text {source }}$. Combining all propagation steps we obtain the input field at the final boundary $\Gamma_{j}$ by

$$
\boldsymbol{V}_{J}^{\text {in }}=P_{J, J-1} \cdot\left(\prod_{i=2}^{J-1} C_{i} \cdot P_{i, i-1}\right) \cdot V_{1}^{\text {source }}
$$

We can choose freely the methods for the individual operators, see Figure 3.

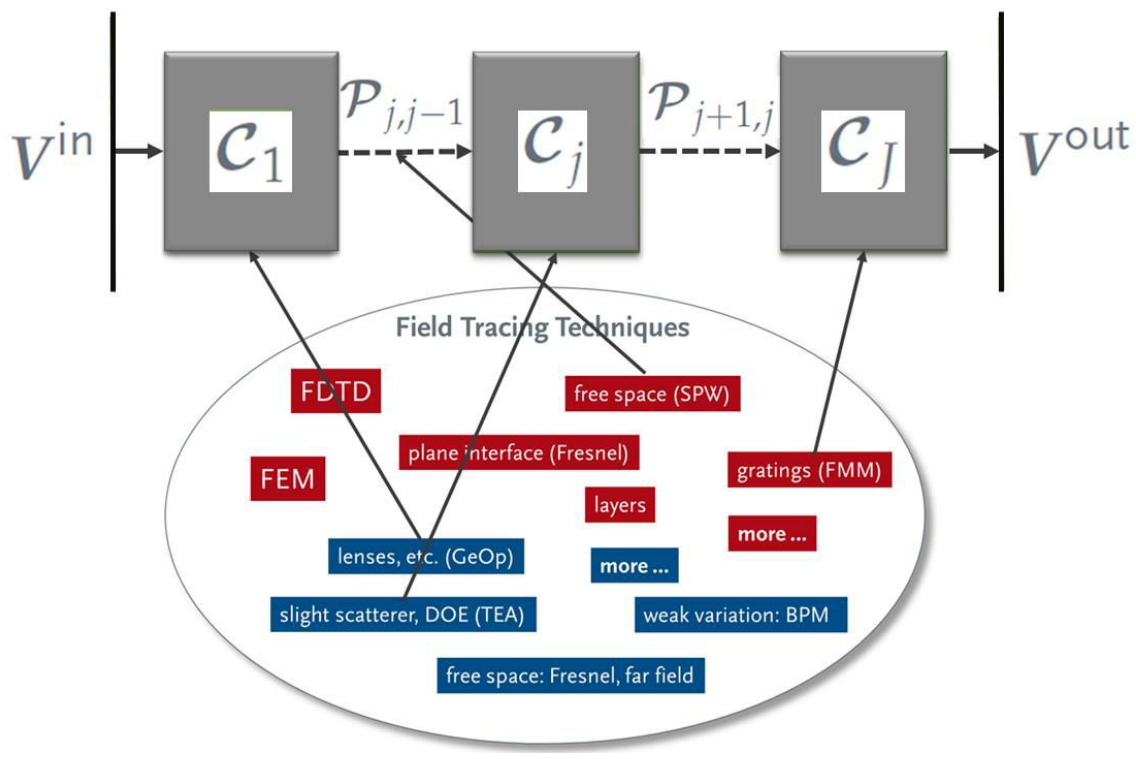

Figure 3: Sequence of operators mapping the input field to the output field.

The operators are chosen such that the relevant physical effects are included whereas the numerical effort is minimal. 


\section{SIMULATION OF AN EYEPIECE}

\subsection{Introduction}

The weight and size of the optical system are critical performance criteria in numerous applications. For instance, the development of hand-holdable long focal length photographic lenses and light head mounted displays (HMD) eyepieces are technical issues of interest.

To get lighter and more compact optical systems, the use of rotationally symmetric diffractive optical elements (DOEs) has become of interest. A rotationally symmetric DOE could be defined by its ideal phase polynomial 4a, by its kinoform and material $4 \mathrm{~b}$ or by its binary $4 \mathrm{c}$ surface and material.

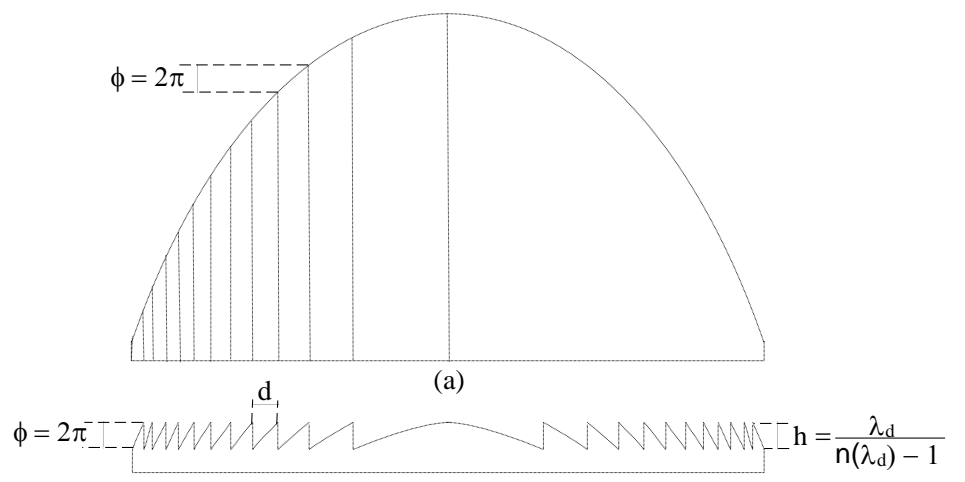

(b)

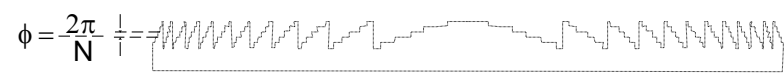

(c)

Figure 4. (a) DOE Ideal phase. (b) Kinoform surface. (c) $\mathrm{N}=5$ levels Binary Surface.

In 2001, Canon introduced the first photographic long focal length lens including a DOE, EF $400 \mathrm{~mm} f: 4$ DO IS [4]. More recently, Zeiss has introduced an OLED HMD including a DOE, Carl Zeiss Cinemizer [5]. The unusual dispersion properties of DOEs make them adequate for the correction of chromatic aberrations. This prevents us from fully achromatizing the optical system by combining different glass materials. This results in a less bulky optical system with fewer lenses.

Unfortunately, the use of DOEs causes stray light due to unwanted orders of diffraction. This makes the use of DOEs less common in practice [5]. The efficiency of the DOE diffraction working order falls below $100 \%$ when waveband width and angles of incidence increase. In 2014, Canon has re-designed the DOE, included in its 400-mm photographic lens, $E F 400 \mathrm{~mm} f: 4$ DO IS II, in order to improve its image quality and decrease its stray light [6].

It appears from the above stated that the use of DOEs is of great interest in the reduction of the height and weight of optical imaging systems; however, the modeling of the stray light coming from the DOEs requires particular attention.

Below, we present the simulation of an eyepiece including a DOE by field tracing methods.

\subsection{Description of the setup}

We consider a visible F/5 and a 40-degree diagonal Field of view (Fov) eyepiece mainly limited by its lateral color, see Figure 5.

In order to improve the eyepiece image resolution, we introduce and optimize a rotationally symmetric DOE between the image plane and the singlet (planar interfaces on the right in Figure 5). Due to the DOE we obtain a correction of lateral 
color. The DOE local zone width, $d$, is smaller with an increasing radial distance. The minimum width is $d=17,5 \mu \mathrm{m}$ at DOE full aperture; whereas, the maximum angle of incidence on the DOE within air does not exceed 22 . The simulation is done with three wavelengths: $486 \mathrm{~nm}, 587 \mathrm{~nm}, 656 \mathrm{~nm}$.
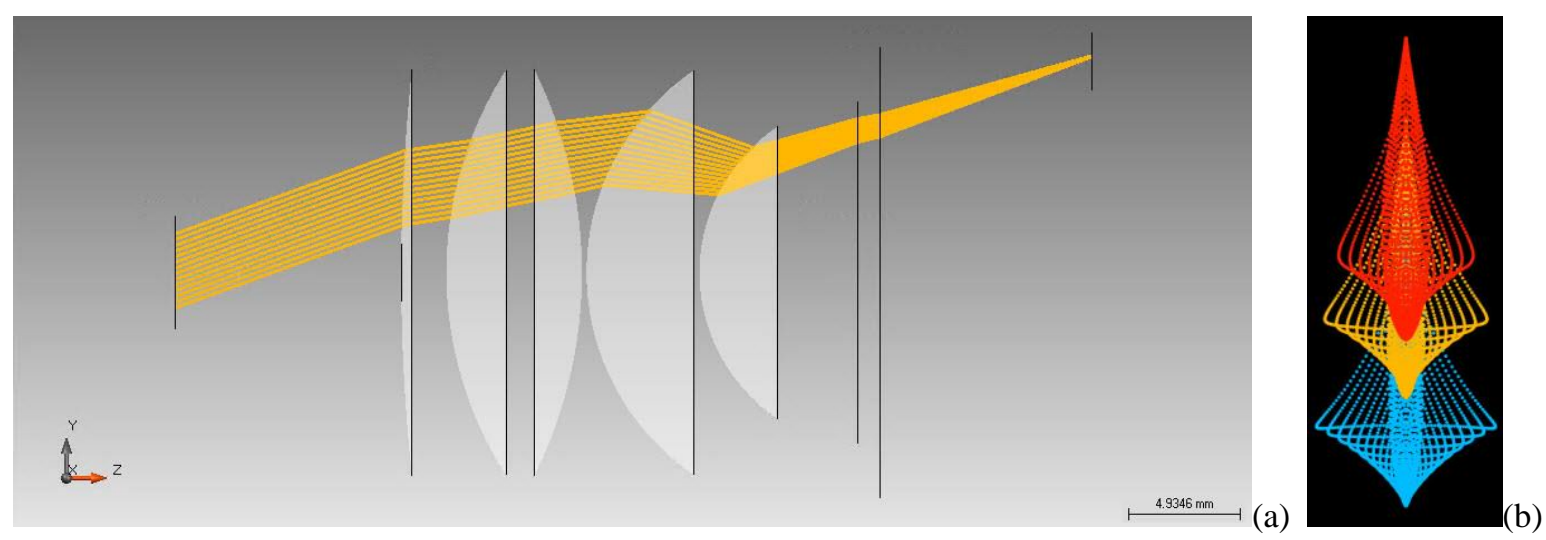

Figure 5. (a) Eyepiece layout with ray tracing at 587nm. (b) Lateral color by spot diagram at 20 degrees computed by field and ray tracing software VirtualLab [3]. Physical height of (b) is $150 \mu \mathrm{m}$

\subsection{Simulation results}

We use the following propagation operators for the field tracing simulation:

- Geometric field tracing for the eyepiece and the PMMA plate of the DOE.

- Spectrum of plane waves (SPW) operator for the free space propagation to the focal region

Both operators have been described in more detail in [1]. The diffractive surface modulation of the DOE causes a phase modulation in the field. Hence, the full information is available and all diffraction orders are considered at once.
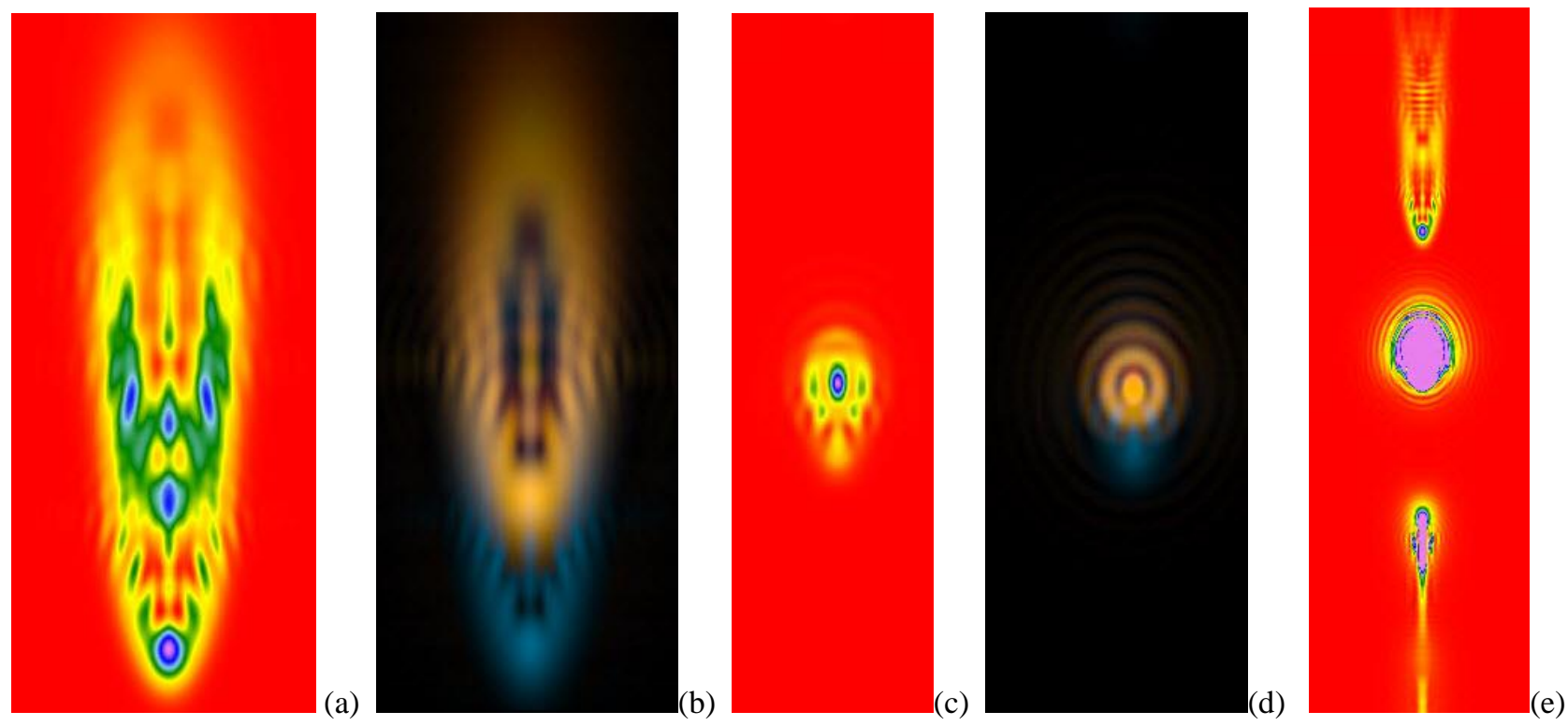

Figure 6. Field Tracing results in the focal region. Physical height of (a)-(d) is $150 \mu \mathrm{m}$, physical height of (e) is $450 \mu \mathrm{m}$. (a)(c)(e) are in false color, (b)(d) are in real color. (a) and (b) show the result for a plate (no DOE). (c) and (d) show the result with DOE. (e) shows the result with DOE including higher orders (over saturation of color scale). Results computed by VirtualLab [3]. 
The use of DOE leads to a focal length decrease and a spot diameter decrease. The results are shown in Figure 6. Indeed the lateral color is reduced considerably by using the DOE (comparison of (a)(b) with (c)(d). The field tracing results show also the higher diffraction orders (see (e)).

\section{CONCLUSION}

We have discussed the simulation of an eyepiece with a diffractive lens with field tracing methods. These methods include the diffraction effects and deliver field information in the focal region of the eyepiece. According to the design target, the reduction of lateral color can be observed. In this paper we have used a geometric field tracing approach for the simulation of the diffractive surface. The accuracy of the simulation can be refined by using a rigorous grating analysis of the local grating. This approach maintains the advantages of field tracing, increases accuracy and may even save computing time since the diffraction orders to be considered can be pre-selected. Such a method is currently under development.

\section{REFERENCES}

[1] Frank Wyrowski and Michael Kuhn. "Introduction to field tracing," Journal of Modern Optics 58(5-6), 449-466 (2011).

[2] Tervo, J.; Turunen, J.; Wyrowski, F. The Light Cube. Fifth EOS Topical Meeting on Advanced Imaging Techniques; European Optical Society: Hannover, 2010; Vol. 3037.

[3] Unified optical design software "Wyrowski VirtualLab Fusion”, developed by Wyrowski Photonics UG, distributed and supported by LightTrans GmbH, Jena, Germany. http://www.lighttrans.com.

[4] Herbert Keppler, "News: How Canon will cut the weight \& size of tele lenses by about one-third." pp 62-63, 148. Popular Photography, Volume 65 Number 1; January 2001. ISSN 0032-4582

[5] Markus Seesselberg and Bernd H. Kleemann, DOEs for Color Correction in Broad Band Optical Systems: Validity and Limits of Efficiency Approximations, Carl Zeiss AG

[6] Stephen Shankland, Canon reveals details for future telephoto lens line, http://www.cnet.com/news/canon-revealsdetails-for-future-telephoto-lens-line/, September 15, 2014 